\title{
THE EXISTENCE OF COMPACT LINEAR MAPS BETWEEN BANACH SPACES
}

\author{
SEYMOUR GOLDBERG AND A. H. KRUSE
}

In [2] J. D. Weston proves that given any separable Banach space $Y$, there exist a normed linear space $X$ and a compact one-one linear operator which maps the conjugate space $X^{\prime}$ onto a subspace dense in $Y$.

It is the purpose of this paper to solve the following problem.

Given normed linear space $X$ and Banach space $Y$, under what conditions does there exist a one-one compact linear map from $X$ onto a subspace dense in $Y$ ?

Necessary and sufficient conditions for such an operator to exist are given (cf. (C) of the theorem below).

We first introduce some notations.

Suppose $X$ and $Y$ are normed linear spaces. Then $B(X, Y)$ (resp., $\mathfrak{H}(X, Y))$ is the space of all bounded (resp., compact) linear maps from $X$ to $Y . B_{0}(X, Y)$ (resp., $\varkappa_{0}(X, Y)$ ) is the set of all one-one maps in $B(X, Y)$ (resp., $\mathfrak{K}(X, Y)) . \bigotimes_{d}(X, Y)$ (resp., $\mathfrak{K}_{d}(X, Y)$ ) is the set of all maps in $\mathbb{B}(X, Y)$ (resp., $\mathfrak{K}(X, Y)$ ) with range dense in $Y . \mathbb{B}_{o, d}(X, Y)$ $=B_{0}(X, Y) \cap \mathbb{B}_{d}(X, Y)$, and $\mathcal{K}_{o, d}(X, Y)=\mathcal{K}_{o}(X, Y) \cap \mathcal{K}_{d}(X, Y)$. Finally, $\varnothing$ is the void set.

If $X$ is a normed linear space and $A$ is a subset of $X^{\prime}$, then $A$ is total if and only if for each $x \neq 0$ in $X$ there exists an $x^{\prime}$ in $A$ such that $x^{\prime} x \neq 0$. The following preliminary remarks are easily verified. The first of these gives alternative ways of stating a condition arising prominently in the rest of the paper.

(i) If $X$ is a normed linear space, then $X^{\prime}$ contains a countable total subset if and only if $X^{\prime}$ is separable with respect to the $w^{*}$ topology and also if and only if $X^{\prime}$ contains a total separable linear subspace.

(ii) If $X$ is a separable normed linear space, then each of the conjugate spaces $X^{\prime}$ and $X^{\prime \prime}$ contains a countable total subset.

(iii) If $X$ and $Y$ are normed linear spaces, $\wp_{o}(X, Y) \neq \varnothing$, and $Y^{\prime}$ has a countable total subset, then $X^{\prime}$ has a countable total subset.

Definition. Suppose $X$ is a Banach space, and suppose $x_{k}$ is in $X$ and $\epsilon_{k}$ is a real number for $k=1,2, \cdots$. Then $\left\{x_{k}\right\}_{1}^{\infty}$ is $\left\{\epsilon_{k}\right\}_{1}^{\infty}$ independent if and only if and

(a) $\sum_{k=1}^{\infty}\left\|\epsilon_{k} x_{k}\right\|<\infty$

Presented to the Society, January 23, 1961 ; received by the editors November 14, 1960 and, in revised form, September 18, 1961. 
(b) for each bounded sequence $\left\{\alpha_{k}\right\}_{1}^{\infty}$ of scalars, $\sum_{k=1}^{\infty} \alpha_{k} \epsilon_{k} x_{k}=0$ implies $\alpha_{k}=0(k=1,2, \cdots)$.

REMARK. $\epsilon_{k} \neq 0(k=1,2, \cdots)$ if $\left\{x_{k}\right\}_{1}^{\infty}$ is $\left\{\epsilon_{k}\right\}_{1}^{\infty}$-independent.

Lemma. Suppose $\left\{x_{k}\right\}_{1}^{\infty}$ is a linearly independent sequence of elements in a Banach space $X$. Then there exists a sequence $\left\{\epsilon_{k}\right\}_{1}^{\infty}$ of positive real numbers such that $\left\{x_{k}\right\}_{1}^{\infty}$ is $\left\{\epsilon_{k}\right\}_{1}^{\infty}$-independent.

PROoF. By considering the sequence $\left\{x_{k} /\left\|x_{k}\right\|\right\}_{1}^{\infty}$, we may assume $\left\|x_{k}\right\|=1(k=1,2, \cdots)$.

For each positive integer $n$, let $l(n)$ be the Banach space of $n$-tuples of scalars with norm defined by $\left\|\left(\eta_{1}, \eta_{2}, \cdots, \eta_{n}\right)\right\|=\sum_{k=1}^{n}\left|\eta_{k}\right|$. The map $f$ defined by $f\left(\eta_{1}, \eta_{2}, \cdots, \eta_{n}\right)=\left\|\sum_{k=1}^{n} \eta_{k} x_{k}\right\|$ is a continuous map from $l(n)$ into the space of real numbers. Define $S_{n}$ to be the set of all $\left(\eta_{1}, \eta_{2}, \cdots, \eta_{n}\right)$ in $l(n)$ such that $1 / 2 \leqq\left\|\left(\eta_{1}, \eta_{2}, \cdots, \eta_{n}\right)\right\| \leqq n$. Then $S_{n}$ is a compact subset of $l(n)$. Hence $f$ attains a minimum $a_{n}$ on $S_{n}$. Since $\left\{x_{k}\right\}_{1}^{\infty}$ is linearly independent, $a_{n}>0$. Clearly, $1>a_{n} \geqq a_{n+1}$ $(n=1,2, \cdots)$.

Let $\epsilon_{1}=1$. Define $\epsilon_{n+1}=2^{-8} \epsilon_{n} a_{n}$ for $n=1,2, \cdots$. Now

$$
0<\epsilon_{n+j} \leqq\left(2^{-8}\right)^{j} \epsilon_{n} a_{n} \leqq 2^{-8 j} \quad(j, n=1,2, \cdots) .
$$

Consider a bounded sequence $\left\{\alpha_{k}\right\}_{1}^{\infty}$ of scalars not all 0 . Let $\alpha$ $=\sup _{k=1}^{\infty}\left|\alpha_{k}\right|$. Then $\alpha>0$, and there exists an integer $N$ such that $\left|\alpha_{N}\right|>\alpha / 2$. Suppose $\left|\epsilon_{n_{0}} \alpha_{n_{0}}\right|=\max _{j=1}^{N}\left|\epsilon_{j} \alpha_{j}\right|$. Then $\left|\epsilon_{n_{0}} \alpha_{n_{0}}\right| \geqq\left|\epsilon_{N} \alpha_{N}\right|$ $>\epsilon_{N} \alpha / 2>0$, and

$$
\begin{aligned}
\left\|\sum_{j=1}^{N} \epsilon_{j} \alpha_{j} x_{j}\right\| & =\left|\epsilon_{n_{0}} \alpha_{n_{0}}\right|\left\|\sum_{j=1}^{N} \epsilon_{j} \alpha_{j} x_{j} /\left(\epsilon_{n_{0}} \alpha_{n_{0}}\right)\right\| \\
& \geqq\left|\epsilon_{n_{0}} \alpha_{n_{0}}\right| a_{N}>\alpha \epsilon_{N} a_{N} / 2 .
\end{aligned}
$$

From (1) we have

$$
\left\|\sum_{j=1}^{\infty} \epsilon_{N+j} \alpha_{N+j} x_{N+j}\right\| \leqq \alpha \sum_{j=1}^{\infty} \epsilon_{N+j} \leqq \alpha \sum_{j=1}^{\infty} 2^{-8 j^{-}} \epsilon_{N} a_{N}<\alpha \epsilon_{N} a_{N} / 4 .
$$

By (2) and (3),

$$
\begin{aligned}
\left\|\sum_{j=1}^{\infty} \epsilon_{j} \alpha_{j} x_{j}\right\| & \geqq\left\|\sum_{j=1}^{N} \epsilon_{j} \alpha_{j} x_{j}\right\|-\left\|\sum_{j=1}^{\infty} \epsilon_{N+j} \alpha_{N+j} x_{N+j}\right\| \\
& >\left(\alpha \epsilon_{N} a_{N} / 2\right)-\left(\alpha \epsilon_{N} a_{N} / 4\right)=\alpha \epsilon_{N} a_{N} / 4>0 .
\end{aligned}
$$

Thus (b) is proved. That (a) holds follows from the fact that

$$
0<\sum_{k=1}^{\infty} \epsilon_{k} \leqq \sum_{j=0}^{\infty} 2^{-8 j}<\infty
$$


Theorem. Suppose $X$ is an infinite-dimensional normed linear space and $Y$ is an infinite-dimensional Banach space. Then (A)-(C) below hold.

(A) $\varkappa_{0}(X, Y) \neq \varnothing$ if and only if $X^{\prime}$ has a denumerable total subset.

(B) $\mathfrak{K}_{d}(X, Y) \neq \varnothing$ if and only if $Y$ is separable.

(C) $\varkappa_{0, d}(X, Y) \neq \varnothing$ if and only if $\mathcal{K}_{0}(X, Y) \neq \varnothing$ (alternatively, $\left.\Theta_{0}(X, Y) \neq \varnothing\right)$ and $\mathfrak{K}_{d}(X, Y) \neq \varnothing$, i.e., if and only if $Y$ is separable and $X^{\prime}$ has a denumerable total subset.

Moreover, each of the sets $\mathfrak{K}_{0}(X, Y), \mathfrak{K}_{d}(X, Y), \mathfrak{K}_{o, d}(X, Y)$ which is nonvoid contains a map which is the limit (in norm) of continuous linear maps having finite-dimensional range.

Proof. There exist linearly independent sequences $\left\{x_{k}^{\prime}\right\}_{1}^{\infty}$ and $\left\{y_{k}\right\}_{1}^{\infty}$ in $X^{\prime}$ and $Y$ respectively such that $\left\|x_{k}^{\prime}\right\|=\left\|y_{k}\right\|=1(k=1,2, \cdots)$. In addition, we may take $\left\{x_{k}^{\prime}\right\}_{1}^{\infty}$ total in $X^{\prime}$ if $X^{\prime}$ has a denumerable total subset, and we may take $\left\{y_{k}\right\}_{1}^{\infty}$ spanning a dense subset of $Y$ if $Y$ is separable. By the lemma, $\left\{x_{k}^{\prime}\right\}_{1}^{\infty}$ is $\left\{\epsilon_{k}\right\}_{1}^{\infty}$-independent and $\left\{y_{k}\right\}_{1}^{\infty}$ is $\left\{\eta_{k}\right\}_{1}^{\infty}$-independent for some $\left\{\epsilon_{k}\right\}_{1}^{\infty}$ and $\left\{\eta_{k}\right\}_{1}^{\infty}$. Let $T: X \rightarrow Y$ and $T_{n}: X \rightarrow Y$ be defined by $T x=\sum_{k=1}^{\infty} \epsilon_{k} \eta_{k} x_{k}^{\prime}(x) y_{k}$ and $T_{n} x$ $=\sum_{k=1}^{n} \epsilon_{k} \eta_{k} x_{k}^{\prime}(x) y_{k}$. Clearly, $T_{n}$ is a bounded linear operator with finite-dimensional range and hence is compact. Moreover, $\left\{T_{n}\right\}_{1}^{\infty}$ converges in norm to $T$, for if $x$ is in $X$ and $\|x\|=1$, then $\left\|T_{n} x-T x\right\|$ $\leqq \sum_{k=n+1}^{\infty} \epsilon_{k} \eta_{k} \rightarrow 0$. Therefore $T$ is a compact linear operator.

Suppose $X^{\prime}$ has a denumerable total subset. Consider $x$ in $X$ such that $T x=0$. Since $\left\{y_{k}\right\}_{1}^{\infty}$ is $\left\{\eta_{k}\right\}_{1}^{\infty}$-independent, $\epsilon_{k} x_{k}^{\prime} x=0$ and hence $x_{k}^{\prime} x=0(k=1,2, \cdots)$. Therefore $x=0$ since $\left\{x_{k}^{\prime}\right\}_{1}^{\infty}$ is total in $X^{\prime}$. Thus $T$ is one-one, $T$ is in $\mathcal{K}_{o}(X, Y)$, and $\mathcal{K}_{o}(X, Y) \neq \varnothing$.

Suppose $Y$ is separable. To show that $(T X)^{-}=Y$, suppose the contrary. Then there exists $y^{\prime} \neq 0$ in $Y^{\prime}$ such that $0=y^{\prime} T X$, whence for each $x$ in $X$,

$$
0=y^{\prime}\left(\sum_{k=1}^{\infty} \epsilon_{k} \eta_{k} x_{k}{ }^{\prime}(x) y_{k}\right)=\sum_{k=1}^{\infty} \epsilon_{k} \eta_{k} y^{\prime}\left(y_{k}\right) x_{k}{ }^{\prime} x=\left(\sum_{k=1}^{\infty} \epsilon_{k} \eta_{k} y^{\prime}\left(y_{k}\right) x_{k}{ }^{\prime}\right)(x) \text {. }
$$

Therefore $\sum_{k=1}^{\infty} \epsilon_{k} \eta_{k} y^{\prime}\left(y_{k}\right) x_{k}^{\prime}=0$. Since $\left\{x_{k}^{\prime}\right\}_{1}^{\infty}$ is $\left\{\epsilon_{k}\right\}_{1}^{\infty}$-independent, it follows that $y^{\prime} y_{k}=0(k=1,2, \cdots)$. Hence $y^{\prime}=0$ since $\left\{y_{k}\right\}_{1}^{\infty}$ generates a subspace dense in $Y$. Thus we have contradicted the statement that $y^{\prime} \neq 0$. Since $(T X)^{-}=Y, T$ is in $\mathcal{K}_{d}(X, Y)$, and $\mathcal{K}_{d}(X, Y) \neq \varnothing$.

If $X^{\prime}$ has a denumerable total subset and $Y$ is separable, then $T$ is in $\mathcal{K}_{。}(X, Y) \cap \mathcal{K}_{d}(X, Y)=\mathcal{K}_{o, d}(X, Y)$, and $\mathcal{K}_{o, d}(X, Y) \neq \varnothing$.

Half of each of (A)-(C) has been proved. The other half of (A) follows from (iii) with the use of (ii) and the observation that for each $T$ in $\mathcal{K}_{\bullet}(X, Y), T(A)$ is separable and $T$ is in $\mathbb{B}_{\bullet}(X, T(A))$. The other 
half of (B) follows from the fact that the range of each map in $\Re(X, Y)$ is separable. The other half of $(\mathrm{C})$ follows from $(\mathrm{A})$ and $(\mathrm{B})$. The last statement of the theorem is now clear from the proof thus far.

Corollary. Suppose $X$ and $Y$ are separable infinite-dimensional normed linear spaces with $Y$ a Banach space. Then $\mathcal{K}_{0, d}(X, Y)$ and $\mathcal{K}_{\bullet, d}\left(X^{\prime}, Y\right)$ are nonempty.

Proof. Apply (ii) and the theorem.

The proof of the theorem leads to the following observations.

ObSERvations. Suppose $X$ is an infinite-dimensional normed linear space and $Y$ is an infinite-dimensional separable Banach space. Then $\left(\mathrm{A}^{\prime}\right)-\left(\mathrm{C}^{\prime}\right)$ below hold.

$\left(\mathrm{A}^{\prime}\right) \mathrm{B}_{\circ}(X, Y) \neq \varnothing$ if and only if $X^{\prime}$ has a denumerable total subset.

(B') $B_{d}(X, Y) \neq \varnothing$.

$\left(C^{\prime}\right) \bigotimes_{0, d}(X, Y) \neq \varnothing$ if and only if $\bigotimes_{\bullet}(X, Y) \neq \varnothing$, i.e., if and only if $X^{\prime}$ has a denumerable total subset.

One could remove "infinite-dimensional" from the hypotheses of the theorem and the observations and still obtain "if and only if" results similar to, but slightly more complicated than, (A)-(C) and $\left(A^{\prime}\right)-\left(C^{\prime}\right)$. The details are completely straightforward.

Statement $\left(\mathrm{A}^{\prime}\right)$ will now be used to generalize a theorem of $\mathrm{J}$. A. Clarkson.

A norm \|\| on a linear space $X$ is called strictly convex if and only if $\|x+y\|<\|x\|+\|y\|$ for all $x$ and $y$ in $X$ which are linearly independent. Suppose $X$ and $Y$ are normed linear spaces with the norm on $Y$ strictly convex, and suppose $T$ is in $B_{0}(X, Y)$. The norm \|\|$^{\prime}$ on $X$ such that $\|x\|^{\prime}=\|x\|+\|T x\|$ for each $x$ in $X$ is strictly convex and is equivalent to the original norm on $X$. Hence, applying $\left(\mathrm{A}^{\prime}\right)$ with $Y$ a Hilbert space, one obtains immediately the following result, whose special case resulting from taking $X$ separable is due to $\mathrm{J}$. A. Clarkson [1, Theorem 9].

Proposition. Suppose $X$ is a normed linear space whose conjugate space has a countable total subset. (For example, suppose $X$ is a separable normed linear space or the conjugate space of a separable normed linear space.) Then the norm of $X$ is equivalent to a strictly convex norm.

\section{REFERENCES}

1. J. A. Clarkson, Uniformly convex spaces, Trans. Amer. Math. Soc. 40 (1936), 396-420.

2. J. D. Weston, A characterization of separable Banach spaces, J. London Math. Soc. 32 (1957), 186-187.

New Mexico State University 